\title{
Pooling of samples to optimize SARS-CoV-2 diagnosis by RT-qPCR: comparative analysis of two protocols
}

\author{
Fabiana Volpato ${ }^{1,2,3}$. Daiana Lima-Morales ${ }^{1,3}$. Priscila Lamb Wink ${ }^{1,3,4}$ - Julia Willig ${ }^{3,5}$. Fernanda de-Paris ${ }^{3}$. \\ Patricia Ashton-Prolla ${ }^{6,7}$. Afonso Luís Barth ${ }^{1,2,3,4}$ (i)
}

Received: 1 September 2020 / Accepted: 9 October 2020 / Published online: 16 October 2020

(C) Springer-Verlag GmbH Germany, part of Springer Nature 2020

\begin{abstract}
This study has aimed to evaluate the use pool of samples as a strategy to optimize the diagnostic of SARS-CoV-2 by RT-qPCR. A total of 220 naso/orofaryngeal swab samples were collected and tested using two different protocols of sample pooling. Results from protocol A were identical with the individual results. However, for results from protocol B, reduced agreement (91\%) was observed in relation to individual testing. Inconsistencies observed were related to RT-qPCR results with higher cycle thresholds. These results suggest that pooling of samples before RNA extraction is preferable in terms of diagnostic for SARS-CoV-2.
\end{abstract}

Keywords Molecular diagnostic $\cdot$ Pooling sample $\cdot$ SARS-CoV-2 $\cdot$ RT-qPCR $\cdot$ Massive testing

\section{Introduction}

Due to the exponential increase of respiratory syndromes by SARS-CoV-2, the World Health Organization (WHO) declared COVID-19 a pandemic of worldwide importance [1]. The rapid propagation of the virus significantly increases the demand on health care system. In response to the challenge of

Afonso Luís Barth

albarth@hcpa.edu.br

1 LABRESIS- Laboratório de Pesquisa em Resistência Bacteriana, Centro de Pesquisa Experimental, Hospital de Clínicas de Porto Alegre (HCPA), Porto Alegre, RS, Brazil

2 Programa de Pós-Graduação em Ciências Médicas (PPGCM), Faculdade de Medicina, Universidade Federal do Rio Grande do Sul (UFRGS), Porto Alegre, RS, Brazil

3 Laboratório de Diagnóstico de SARS-CoV-2 (Lab Covid), Hospital de Clínicas de Porto Alegre (HCPA), Porto Alegre, RS, Brazil

4 Programa de Pós-Graduação em Ciências Farmacêuticas (PPGCF), Faculdade de Farmácia, UFRGS, Porto Alegre, RS, Brazil

5 Programa de Residência Multiprofissional do Hospital de Clínicas de Porto Alegre (HCPA), Porto Alegre, RS, Brazil

6 Departamento de Genética, UFRGS, Porto Alegre, RS, Brazil

7 Grupo de Pesquisa e Pós-Graduação e Pós-Graduação, HCPA, Porto Alegre, RS, Brazil identifying and isolating infected patients, two different types of tests have been used: real-time reverse transcriptase polymerase chain reaction (RT-qPCR) and serum IgM and/or IgG antibody detection assays.

In order to avoid diagnostic cross-reactivity with other coronaviruses, most RT-qPCR assays include primers for at least two different molecular targets, such as nucleocapsid proteins $1(\mathrm{~N} 1)$ and 2 (N2) [2]. Also, this procedure could minimize false-negatives results associated with difference in sensitivity of primers. A major advantage of real time assays is that amplification and analysis of the final product are performed simultaneously in a closed system.

Several countries have used RT-qPCR in large-scale efforts as a mainstream initiative for combating the SARS-CoV-2 pandemic. To enable massive testing, thousands of laboratories worldwide have expanded their routine and high-throughput automated testing was implemented mainly in reference centers. However, many laboratories, especially in underserved areas, became overloaded and new approaches to cope with this ever increasing and long-lasting challenge are needed. A few studies have provided encouraging results that support the use of sample pooling as a robust and cost-/time-saving approach in this extreme scenario. In this brief report, we provide additional evidence in favor of SARS-CoV-2 RT-qPCR analysis with pooled samples using two different methodologies. 


\section{Methods}

A total of 220 samples from different patients were obtained by oro/nasopharyngeal swabbing performed by trained personnel in a single institution. Individual swabs were mixed with $3 \mathrm{~mL}$ of $\mathrm{NaCl} 0.9 \%$ solution. Two strategies of pooling samples were evaluated as follows: (1) protocol A: consisted of 10 samples grouped $(100 \mu \mathrm{L}$ from each sample) before RNA extraction; (2) protocol B: consisted of 10 samples group $(4 \mu \mathrm{L}$ of each) after individual RNA extraction. Results from both protocols were compared to a reference protocol (protocol R): individual analyses of the same samples, performed in parallel to the analysis of the pools. RNA was extracted from a volume of $600 \mu \mathrm{L}$ of respiratory specimen using the Abbott m2000 instrument (Abbott Laboratories, USA) which yielded $80 \mu \mathrm{L}$ of nucleic acid in the eluted buffer.

A previously validated SARS-CoV-2 RT-qPCR protocol following the $\mathrm{CDC}$ guidelines and primers was used. In brief, Superscript III (SSIII) one-step RT-qPCR system (Thermo Fisher Scientific Inc., USA) was used for reactions. Master mix was composed of $5 \mathrm{uL}$ of $2 \mathrm{X}$ reaction buffer $(0.4 \mathrm{mM}$ of each dNTP and $\left.6 \mathrm{mM} \mathrm{MgSO}_{4}\right) ; 0.2 \mu \mathrm{L}$ of SuperScript ${ }^{\mathrm{TM}} \mathrm{III}$ RT/Platinum ${ }^{\mathrm{TM}}$ Taq Mix; $0.2 \mu \mathrm{L}$ of ROX (1:10); $0.75 \mu \mathrm{L}$ of combined primers/probes mix of nCOV1 (N1 primer) or nCOV2 (N2 primer) or RP (2019-nCoV RUO Kit, Integrated DNA Technologies Inc., USA); and $4 \mu \mathrm{L}$ of RNA. The human ribonuclease P gene (RNAse-P) was used as internal control to monitor nucleic acid extraction, specimen quality, and presence of inhibitors. Thermal cycling was performed at $50{ }^{\circ} \mathrm{C}$ for $30 \mathrm{~min}$ for reverse transcription, followed by $95{ }^{\circ} \mathrm{C}$ for 2 min and 45 cycles of $95{ }^{\circ} \mathrm{C}$ for $15 \mathrm{~s}, 55{ }^{\circ} \mathrm{C}$ for $35 \mathrm{~s}$ in QuantStudio ${ }^{\circledR} 3$ Applied Biosystems $^{\mathrm{TM}}$ (Applied Biosystems, USA). The result was considered "negative" when neither N1 nor N2 targets amplified and "positive" when both N1 and N2 amplified. RTqPCR was considered "inconclusive" when only one target (N1 or N2) amplified. Individual samples and pools with positive results had the values of the cycle threshold $(\mathrm{Ct})$ recorded and compared. To minimize inter-experiment variability ("batch effects"), simultaneous analysis of individual samples and their corresponding pools was undertaken at same RTqPCR reaction.

\section{Results}

The 220 samples included in this study were sorted in 22 pools with 10 clinical samples each, for each protocol. The individual composition of the 22 pools for $\mathrm{A}$ and $\mathrm{B}$ was as follows: all negative samples $(1 / 22)$, one inconclusive sample $(1 / 22)$, only one positive sample $(8 / 22)$, two positive samples $(7 / 22)$, three positive samples ( $3 / 22)$, four positive samples $(1 / 22)$, and five positive samples (1/22) (Table 1). There was $100 \%$ and $91 \%$ of agreement when the results from individual and pooled samples with protocol A (22 of 22) and protocol B (20 of $22)$, respectively. The $\mathrm{Cts}$ of the individual samples with positive results ranged from 13.22 to 37.14 , for both $\mathrm{N} 1$ and N2 targets. The Cts of two individual samples with divergent results in protocol B were $>32.73$ - Table 1. RNAse-P used as a quality control presented very satisfactory results (Cts between 26 and 32).

It was possible to observe that Cts (both targets) of pooled samples in protocol A with positive results in RT-qPCR tended to be 3 units higher in average when compared to Cts of protocol R (Table 1). This increase was also observed in pools of the protocol B for the N1 target; but the Ct of N2 target presented a difference of 4 units in average compared to protocol R. Noteworthy, in pools with more than one positive sample, $\mathrm{Ct}$ of the pool was similar to the lowest $\mathrm{Ct}$ obtained in the individual samples included in the pool (Table 1).

\section{Discussion}

A rapid and reliable laboratory diagnosis of SARS-CoV-2 infection plays a crucial role for public health interventions related to the new coronavirus pandemic [3]. RT-qPCR detection of the virus by oro/nasopharyngeal swabbing is characterized by good sensitivity and high specificity and has been regarded as the "gold standard" for the virus genome detection [4]. However, it is still a semi-automated strategy which includes several sequential analytical steps and is associated with significant costs. Sample pooling is well-known approach to optimize time and costs of massive screening strategies for infectious diseases and has been reported as a reliable technique for hepatitis $\mathrm{B}$, hepatitis $\mathrm{C}$, and human immunodeficiency virus in blood banks [5].

In this study, we demonstrate that RT-qPCR with primers for the nucleocapsid of SARS-CoV-2 using pooled samples (10/pool) is able to provide a correct diagnosis even when only one individual sample is positive in the pool. In fact, the pool RT-qPCR analysis identified a positive sample (protocol A) even when the single sample analysis had a later $\mathrm{Ct}$ $(\mathrm{Ct}>32.73)$. Therefore, according to our experience, we suggest to make pools with a maximum of 10 samples/pool as this approach increases significantly the diagnostic capacity of the labs without losing quality.

However, the size of the pool can accommodate different infection scenarios and it can be adequate if necessary, according to the institution necessity as suggested by Lohse et al [6]. It has to be considered that the use of pools would present best performance whether the population in analysis is expected to present low incidence of the COVID-19 such as people without symptoms. In fact, the pooling strategy would save time and resources when massive testing is required as long as the 
Table 1 Cycle threshold results of pooling samples for detection of SARS-CoV-2 by RT-qPCR

\begin{tabular}{|c|c|c|c|c|c|c|c|}
\hline $\begin{array}{l}\text { Pool } \\
\text { identification }\end{array}$ & $\begin{array}{l}\text { Protocol } \\
\text { A* Ct N1 }\end{array}$ & $\begin{array}{l}\text { Protocol } \\
\text { A Ct N2 }\end{array}$ & $\begin{array}{l}\text { Protocol } \\
\mathrm{B} * * \mathrm{Ct} \mathrm{N} 1\end{array}$ & $\begin{array}{l}\text { Protocol } \\
\text { B Ct N2 }\end{array}$ & $\begin{array}{l}\text { Number of positive } \\
\text { samples in the pool }\end{array}$ & $\begin{array}{l}\text { Value of lower Ct } \\
\text { positive in the pool N1 }\end{array}$ & $\begin{array}{l}\text { Value of lower Ct } \\
\text { positive in the pool N2 }\end{array}$ \\
\hline Pool 1 & Negative & Negative & Negative & Negative & 0 & - & - \\
\hline Pool 2 & 35.44 & 38.46 & Negative & Negative & 1 & 32.73 & 35.05 \\
\hline Pool 3 & 22.02 & 22.64 & 22.26 & 22.99 & 2 & 19.02 & 19.96 \\
\hline Pool 4 & 16.66 & 16.82 & 16.84 & 16.78 & 1 & 13.54 & 13.22 \\
\hline Pool 5 & 20.57 & 20.98 & 20.96 & 21.25 & 1 & 17.39 & 17.85 \\
\hline Pool 6 & Negative & 27.72 & Negative & Negative & Inconclusive & Negative & 37.14 \\
\hline Pool 7 & 24.13 & 25.31 & 27.36 & 29.06 & 1 & 20.37 & 20.23 \\
\hline Pool 8 & 35.41 & 38.97 & Negative & 38.42 & 1 & 33.79 & 36.28 \\
\hline Pool 9 & 19.35 & 21.18 & 19.81 & 21.53 & 2 & 16.06 & 17.39 \\
\hline Pool 10 & 21.04 & 21.94 & 20.69 & 20.59 & 2 & 18.17 & 18.82 \\
\hline Pool 11 & 21.61 & 23.29 & 21.48 & 23.42 & 5 & 19.12 & 18.91 \\
\hline Pool 12 & 31.21 & 33.27 & 30.41 & 33.23 & 2 & 27.07 & 29.23 \\
\hline Pool 13 & 20.54 & 20.32 & 24.48 & 25.12 & 4 & 20.89 & 20.55 \\
\hline Pool 14 & 24.08 & 27.24 & 20.79 & 22.36 & 3 & 18.23 & 19.39 \\
\hline Pool 15 & 17.00 & 18.21 & 16.97 & 18.18 & 3 & 13.55 & 15.01 \\
\hline Pool 16 & 28.89 & 31.42 & 28.81 & 31.23 & 2 & 25.47 & 27.17 \\
\hline Pool 17 & 20.50 & 21.41 & 20.96 & 21.96 & 3 & 17.97 & 18.74 \\
\hline Pool 18 & 27.57 & 29.11 & 26.54 & 28.7 & 2 & 24.05 & 25.12 \\
\hline Pool 19 & 21.34 & 22.06 & 21.41 & 22.40 & 1 & 18.13 & 18.59 \\
\hline Pool 20 & 32.61 & 34.17 & 32.54 & 39.07 & 1 & 29.33 & 30.51 \\
\hline Pool 21 & 28.02 & 29.26 & 26.99 & 27.26 & 2 & 23.68 & 24.26 \\
\hline Pool 22 & 19.23 & 19.73 & 19.31 & 19.76 & 1 & 16.11 & 16.74 \\
\hline
\end{tabular}

*Protocol A: Pooling of clinical samples prior to perform RNA extraction

**Protocol B: Pooling samples after individual RNA extraction

vast majority of people are asymptomatic. However, the use of pools would not be an adequate strategy when testing individuals with high pretest probability of the disease.

The best performance of protocol A in relation to protocol $\mathrm{B}$ in our study is probably related to a larger volume of each individual clinical sample added to the pool. Furthermore, protocol A is operationally straightforward and less time consuming as it requires only one RNA extraction. Moreover, the only pool which contained an individual sample with inconclusive result presented inconclusive result only in protocol A.

According to Buckingham et al [7], the absolute Ct comparison is only meaningful when comparing experiments using the same reaction conditions. In this study, the individual and pooled samples were tested together in same experimental run, and using same reagents. Considering the $\mathrm{Ct}$ differences between a positive individual sample and the same sample tested in a pool, we observed an average variation of 3 units of $\mathrm{Ct}$ (protocol A). The $\mathrm{Ct}$ loss when pooling is justified by the fact that the positive sample is diluted in the pool. According to the literature, using the same sample in a 10-fold dilution series, the $\mathrm{Ct}$ values differ by $\sim 3.3$ units [7]. These data corroborate with the $\mathrm{Ct}$ variation found in this study between the result of RT-qPCR of the individual sample and of this sample in a pool with nine others. We see as limitations of our study the fact that we used only the US CDC protocol and that the number of pools evaluated is low.

Finally, the results of this study indicate that it is possible to obtain positive results of RT-qPCR when combining 10 clinical samples in one pool (protocol A). The pooling of clinical samples would increase significantly test capacity of the laboratories. The use of pools would be much more effective to test clinical samples in scenarios of low prevalence of SARSCoV-2 as a positive result of the pool will require that all samples be tested individually. In conclusion, to test populations in a large scale, the use of pools is a very useful strategy as an epidemiological screening method.

Funding This study received a grant of "Fundação de Apoio a Pesquisa e Ensino do rio Grande do Sul (FAPERGS)" (\#20-2551-0000265-9) as well as of "Fundo de Incentivo a Pesquisa e Eventos do Hospital de Clínicas de Porto Alegre (FIPE/HCPA) - (\#2020-0163).

Data availability The datasets generated during and/or analyzed during the current study are available from the corresponding author on reasonable request. 


\section{Compliance with ethical standards}

Conflict of interest The authors declare that they have no conflicts of interests.

Ethical approval This work has the approval of the ethics committee under the number C.A.A.E.: 30767420.2.0000.5327 of Porto Alegre Clinical Hospital.

Consent to participate Not applicable.

Consent to publication Not applicable.

\section{References}

1. Sun J, He WT, Wang L, Lai A, Ji X, Zhai X, Li G, Suchard MA, Tian J, Zhou J, Veit M, Su S (2020) COVID-19: epidemiology, evolution, and cross-disciplinary perspectives. Trends Mol Med 26:483-495. https://doi.org/10.1016/j.molmed.2020.02.008

2. Tang YW, Schmitz JE, Persing DH, Stratton CW (2020) Laboratory diagnosis of COVID-19: current issues and challenges. J Clin Microbiol 58:e00512-e00520. https://doi.org/10.1128/JCM.00512-20
3. Lu X, Whitaker B, Sakthivel SKK, Kamili S, Rose LE, Lowe L, Mohareb E, Elassal EM, Al-sanouri T, Haddadin A, Erdman DD (2014) Real-time reverse transcription-PCR assay panel for Middle East respiratory syndrome coronavirus. J Clin Microbiol 52(1):67-75. https://doi.org/10.1128/JCM. 02533-13

4. Shen M, Zhou Y, Ye J, Al-maskri AAA, Kang Y, Zeng S, Cai S (2020) Recent advances and perspectives of nucleic acid detection for coronavirus. J Pharm Anal 10:97-101. https://doi.org/10.1016/j. jpha.2020.02.010

5. Nguyen T, Bang DD, Wolff A (2020) 2019 novel coronavirus disease (COVID-19): paving the road for rapid detection and point-ofcare diagnostics. Micromachines 11. https://doi.org/10.3390/ mi11030306

6. Lohse S, Pfuhl T, Berkó-Göttel B, Rissland J, Geißler T, Gärtner B (2020) Pooling of samples for testing for SARS-CoV-2 in asymptomatic people. Lancet Infect Dis. https://doi.org/10.1016/S14733099(20)30362-5

7. Buckingham L (2019) Chapter 6: nucleic acid amplification. In Buckingham L. Molecular diagnostics: Fundamentals, methods, and clinical applications, 3rd edn. F.A. Davis Company, Philadelphia, p 142-178

Publisher's note Springer Nature remains neutral with regard to jurisdictional claims in published maps and institutional affiliations. 